University of Nebraska - Lincoln

DigitalCommons@University of Nebraska - Lincoln

$12-1-1997$

\title{
Freezing of Confined Water: A Bilayer Ice Phase in Hydrophobic Nanopores
}

\author{
Kenichiro Koga \\ University of Nebraska-Lincoln, koga@cc.okayama-u.ac.jp \\ Xiao Cheng Zeng \\ University of Nebraska-Lincoln, xzeng1@unl.edu \\ Hideki Tanaka \\ Kyoto University, htanakaa@cc.okayama-u.ac.jp
}

Follow this and additional works at: https://digitalcommons.unl.edu/chemzeng

Part of the Chemistry Commons

Koga, Kenichiro; Zeng, Xiao Cheng; and Tanaka, Hideki, "Freezing of Confined Water: A Bilayer Ice Phase in Hydrophobic Nanopores" (1997). Xiao Cheng Zeng Publications. 67.

https://digitalcommons.unl.edu/chemzeng/67

This Article is brought to you for free and open access by the Published Research - Department of Chemistry at DigitalCommons@University of Nebraska - Lincoln. It has been accepted for inclusion in Xiao Cheng Zeng Publications by an authorized administrator of DigitalCommons@University of Nebraska - Lincoln. 


\title{
Freezing of Confined Water: A Bilayer Ice Phase in Hydrophobic Nanopores
}

\author{
Kenichiro Koga, ${ }^{1}$ X. C. Zeng, ${ }^{1}$ and Hideki Tanaka ${ }^{2}$ \\ ${ }^{1}$ Department of Chemistry and Center for Materials Research and Analysis, University of Nebraska, Lincoln, Nebraska 68588 \\ ${ }^{2}$ Department of Polymer Chemistry, Kyoto University, Kyoto, 606-01 Japan
}

(Received 30 July 1997)

\begin{abstract}
Molecular dynamics simulations were performed to study the phase behavior of a thin film of water confined to a slit nanopore with smooth walls. A first-order water-to-ice freezing transition has been observed. The resulting ice, which is a crystal of bilayer consisting of rows of distorted hexagons, does not resemble any ice crystals found so far. The confined water contracts upon freezing when the confinement load is low $(\sim 0.5 \mathrm{kbar})$ and expands when the load is high (10 kbar). The residual entropy of the bilayer ice can be calculated exactly, which is about half of the entropy of the bulk ice. [S0031-9007(97)04905-3]
\end{abstract}

PACS numbers: 61.20.Ja, 61.20.Qg, 68.15.+e, 92.40.Sn

Normal water possesses a high freezing point and expands as it freezes. The origin of these anomalies can be traced to the ability of water molecules to associate themselves via hydrogen bonding. When confined in nanopores the properties of water can be dramatically different from their bulk counterpart. For example, Porter and ZinnWarner [1] have reported that Young's modulus of solidwater thin films reaches its maximum value of $\sim 20 \%$ of the bulk value as increasing film thickness from 0.5 to $0.8 \mathrm{~nm}$, implying the possibility of confinement-driven order-disorder phase transition. However, the phase behavior of water confined in nanopores remains largely unexplored. Here we report the first simulation results of water-to-ice freezing transition in a nanopore along with the discovery of a new ice phase.

We performed molecular dynamics (MD) simulations at a fixed temperature $(T)$ and fixed load $\left(P_{z z}\right.$; the normal pressure) on two plane-parallel solid walls [2,3], which confine a thin film (at the nanoscale) of water. The system consists of a fixed number $(N)$ of water molecules $(N=192)$. The water molecules interact with each other via the TIP4P potential [4-6] and with the walls via the 9-3 Lennard-Jones potential [7]. The long-range waterwater intermolecular potential is smoothly truncated at $8.7 \AA$. The simulation cell is a rectangular prism (lamella) with lateral dimensions $l_{x}=32.51 \AA$ and $l_{y}=28.15 \AA$, which is sufficiently large that properties of the system at the fixed area density $\left(\rho_{A}=N / l_{x} l_{y}\right)$ do not depend on the cell size or shape [8]. Periodic boundary conditions are imposed in the $x$ and $y$ directions. For a given temperature and load, the simulation time ranges from 1.2 to $11 \mathrm{~ns}$. Normal loads of $50 \mathrm{MPa}, 150 \mathrm{MPa}$, and $1 \mathrm{GPa}$ were applied in three different simulations. At $340 \mathrm{~K}$, the load lower than $50 \mathrm{MPa}$ is found to be insufficient for maintaining a stable liquid phase in the hydrophobic slit pore. For each fixed load, the temperature is first lowered in steps from 340 to $180 \mathrm{~K}$ and is then raised in steps from the freezing point until the melting is completed.

Figure 1 shows the temperature dependence of the potential energy, excluding contributions from the water- wall interactions and subtracting the mean potential energy of the harmonic oscillators $3 R T$, where $R$ is the gas constant. As the system is cooled, the potential energy first gradually decreases with a slightly higher rate than in bulk water [9] and then suddenly drops by about $5 \mathrm{~kJ} / \mathrm{mol}$ to a lower value. Upon heating, the potential energy jumps to a higher value at a temperature higher than the transition temperature in the cooling process. The discontinuity and the strong hysteresis shown in the potential energy curves suggest a first-order phase transition. The mean square displacement $\left\langle r^{2}(t)\right\rangle$ in the hightemperature phase increases linearly with time, whereas $\left\langle r^{2}(t)\right\rangle$ in the low-temperature phase oscillates with a mean slope of nearly zero, indicating that the two phases are liquid and solid, respectively.

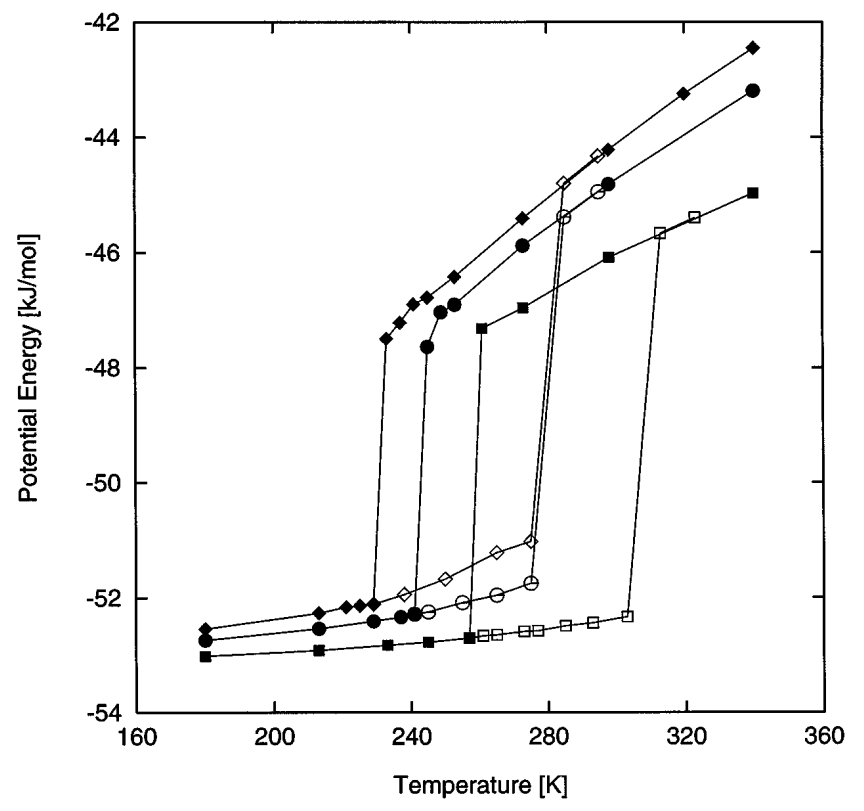

FIG. 1. Temperature dependence of potential energies at normal loads of $50 \mathrm{MPa}$ (diamond), $150 \mathrm{MPa}$ (circle), and $1 \mathrm{GPa}$ (square). The filled and unfilled marks indicate the cooling process and heating process, respectively. 
A question naturally arises: Is the solid an amorphous ice [9-12] or a crystalline ice? To answer this question, one needs to analyze the structure of the solid phase. Every instantaneous configuration generated in the MD simulation has a vibrational displacement from a local minimum of the potential energy hypersurface [13]. To remove the vibrational displacement we applied the steepest descent method; the resulting configurations were used for the structural analyses.

Figures 2(a)-2(c) display top and side views of the ice phase, and a top view of the liquid phase under $1 \mathrm{GPa}$ load. The solid is a bilayer ice crystal. We also confirmed that the solid phase that occurs under the lower loads (50 and $150 \mathrm{MPa}$ ) has the same crystalline structure. It appears that the structure of this bilayer ice crystal resembles none of the structures of existing ice polymorphs nor those of ices found in metallic or hydrophilic pores [1,14-17]. The top view of the bilayer [Fig. 2(a)] shows a distorted hexagonal lattice structure. The upper and lower hexagonal lattices are completely in registry. Unlike the puckered hexagonal layers of ice Ih, each layer of the bilayer ice is almost flat, that is, all the oxygen atoms in each layer are located in a common plane [Fig. 2(b)]. Nevertheless, the bilayer ice has a feature that is common to all known ice polymorphs [18]: Every water molecule is hydrogen bonded to its four neighbors. Thus the hydrogen-bonding network of the bilayer ice is completely self-closed within the bilayer even though it is considerably distorted from the tetrahedral geometry of ice Ih. Note that this freezing transition is very sensitive to the value of $\rho_{A}$ : Half or double the value of $\rho_{A}$ will not lead to the bilayer ice [8].

The hydrogen-bonding network of the distorted hexagonal layer can be characterized as follows; First, all of the hexagons having oxygen atoms at the vertices are congruent but with two different chiralities (we call them $A$ and $B$ ). Half of the hexagons have chirality $A$ and half chirality $B$. Each hexagon has a side length (i.e., the nearest neighbor $\mathrm{O}-\mathrm{O}$ separation) of $2.73 \pm 0.02 \AA$ and three different angles (i.e., the O-O-O angles): $\alpha=108^{\circ}$, $\beta=118.5^{\circ}$, and $\gamma=133.5^{\circ}$ (all within error bar $\pm 2^{\circ}$ ). The latter two angles are much larger than the $\mathrm{HOH}$ bond angle $104.5^{\circ}$ of water. In fact, we never observe that the water molecules are so oriented that the $\mathrm{HOH}$ bond angle is superposed over the $\beta$ or $\gamma$ angles; we refer this as the first bilayer ice rule. Second, when one arm of a water molecule is normal to the hexagonal lattice plane, the other arm never points towards direction OP [see the inset of Fig. 3(a)]; we refer to this as the second bilayer ice rule. Third, hexagons with the same chirality (e.g., $A$ ) always line up in a row. Finally, the bilayer ice is composed of alternating rows of $A$ 's and $B$ 's $(A B A B$ stacking).

We note that, under the two bilayer ice rules, the total number of the possible arrangements of molecular orientation can be counted exactly, which is $W^{\text {bilayer }}=2^{N / 4}$ (a)

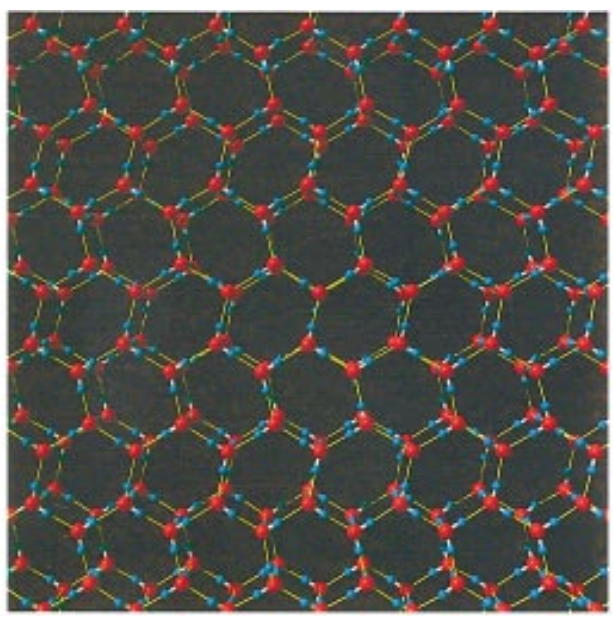

(b)

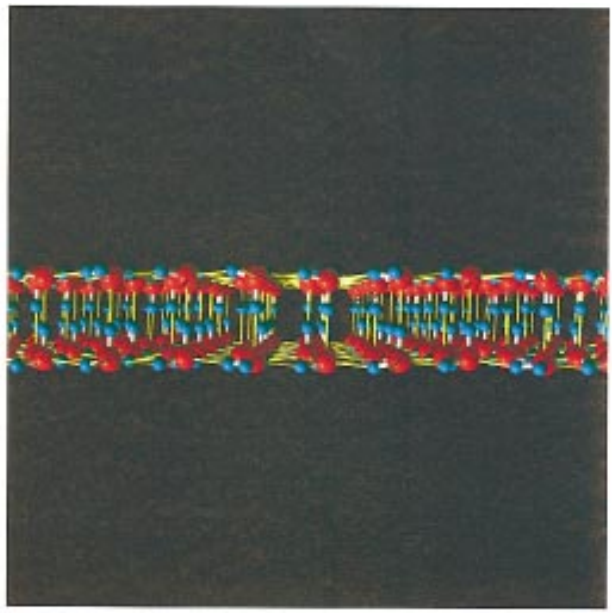

(c)

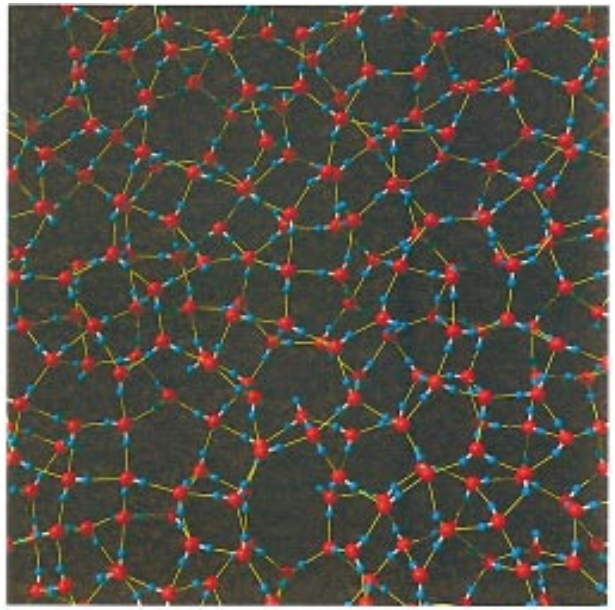

FIG. 2(color). Structure of the thin film of water: (a) solid phase top view; (b) solid phase side view, (c) liquid phase top view. The vibrational displacement from the inherent structure is removed, by using the steepest descent method, from an instantaneous structure at $180 \mathrm{~K}$ (solid phase) and at $298 \mathrm{~K}$ (liquid phase). These structures are obtained under the load of $1 \mathrm{GPa}$. Essentially the same crystal structure is obtained under the loads of 50 and $150 \mathrm{MPa}$. (Image molecules are also drawn in the picture, and the boundary of the simulation cell is not parallel to the boundary of the top view.) 

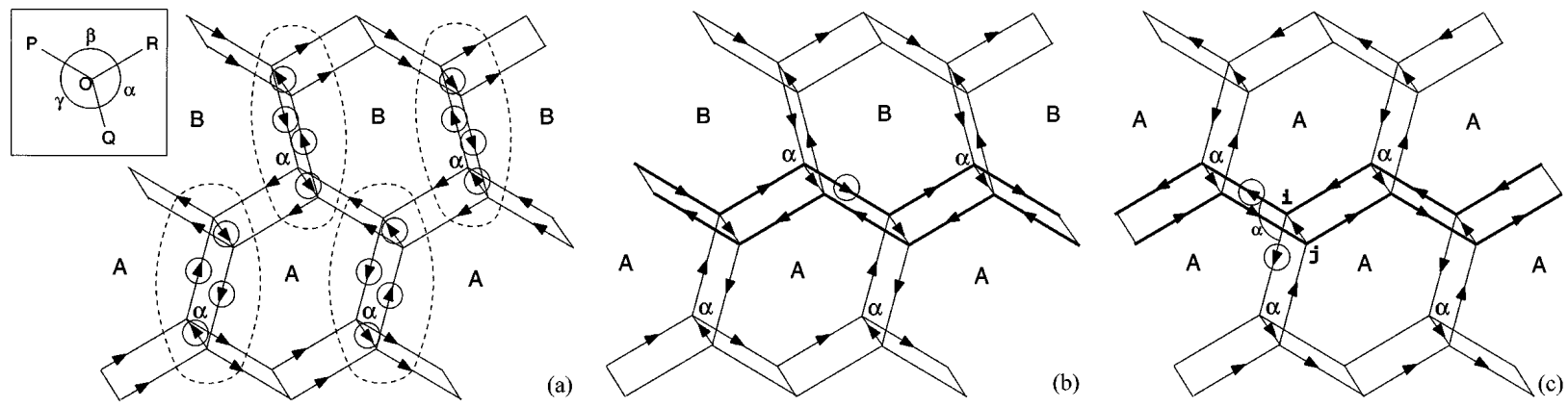

FIG. 3. (a) Schematic diagram of a possible arrangement of molecular orientations in a portion of the bilayer ice crystal. Oxygen atoms are located at vertices of hexagons and directions of the $\mathrm{OH}$ arms are denoted by the arrows. The inset shows the directions of three neighbors with respect to a molecule located at $\mathrm{O}$. In the bilayer ice, no water molecule points its $\mathrm{OH}$ arms towards the direction OP (the second bilayer ice rule). Therefore all of the directions of the $\mathrm{OH}$ arms, denoted by the arrows without a circle, are uniquely determined. Let us focus on a set of four water molecules surrounded by the dashed curve. Among eight OH arms, directions of four $\mathrm{OH}$ arms are uniquely determined; one possible arrangement of the other four $\mathrm{OH}$ arms is represented by the arrows with a circle. Besides this arrangement, there is only one other possible arrangement for the latter four $\mathrm{OH}$ arms, represented by inverting the directions of the four arrows in the circles simultaneously. Thus there are only two possible arrangements for each set surrounded by the dashed curve. Since the two arrangements for each set are independent of arrangements of other sets, the total number of the possible arrangements for the crystal having $N$ molecules is given by $2^{N / 4}$. (b) An arrangement of OH arms such that the second bilayer ice rule is violated, as shown by the arrow in the circle. As a result, directions of other $\mathrm{OH}$ arms denoted by arrows are uniquely determined. Thus there are only two possible arrangements for molecules in the boundary (thick lines) separating the two rows. Since the number of molecules in the boundary is of the order of $\sqrt{N}$, the total number of the possible arrangements for a bilayer crystal containing such a boundary becomes smaller by a factor of $2^{-\sqrt{N} / 4}$. (c) An arrangement of $\mathrm{OH}$ arms in successive rows of the same kind (e.g., $A A$ ). Let us focus on a pair of molecules $i$ and $j$. One of them must be oriented so that its bond angle is superposed over the $\alpha$ angle, according to the first bilayer ice rule. If the bond angle of molecule $i$ is superposed over the $\alpha$ angle, as shown by arrows in circles, all the directions of OH arms shown by other arrows are uniquely determined due to the first rule. Thus there are only two possible arrangements for molecules in the boundary (thick lines) separating the two rows. Again, since the number of molecules in the boundary is of the order of $\sqrt{N}$, the total number of the possible arrangements becomes smaller by a factor of $2^{-\sqrt{N} / 4}$. In particular, the $A A A A$ stacking crystal has a residual entropy of $k_{B} \ln 2$.

[see Fig. 3(a)]. Thus the residual entropy of the bilayer ice crystal is given by

$$
S^{\text {bilayer }}=k_{B} \ln W^{\text {bilayer }}=k_{B} \ln 2^{N / 4},
$$

where $k_{B}$ is the Boltzmann constant. Note that $2^{N / 4}$ is smaller than $(3 / 2)^{N}$, the number for ice Ih (first estimated by Pauling [19] and later shown, by Onsager and Dupuis [20], to be a lower bound of the actual number). Therefore the residual entropy of the bilayer ice is less than half of the residual entropy of ice Ih: $S^{\text {bilayer }}=$ $1.44 \mathrm{~J} \mathrm{~mol}^{-1} \mathrm{~K}^{-1}$ and $S^{\text {icelh }}=3.37 \mathrm{~J} \mathrm{~mol}^{-1} \mathrm{~K}^{-1}$.

Can the two bilayer ice rules be violated? For the first one it is very unlikely because it would be energetically very unfavorable for the $\mathrm{HOH}$ bond angle to be superposed over the $\beta$ or $\gamma$ angles. On the other hand, the second ice rule seems not as strict as the first one although violation of the second rule has not been found in the MD simulations. To shed more light on this question, we build a bilayer ice crystal such that a molecular orientation arrangement violates the second rule [see Fig. 3(b)]. Interestingly, we find that this violation always leads to a smaller residual entropy and a higher potential energy. Indeed, our calculation shows that the potential energy can be $2 \mathrm{~kJ} \mathrm{~mol}^{-1}$ higher when the molecular orientation arrangement violates the second rule to the highest degree. Moreover, we find that other ways of stacking $A$ and $B$ rows (e.g., the $A A A A$ stacking, or a random mixture of $A$ and $B$ rows),

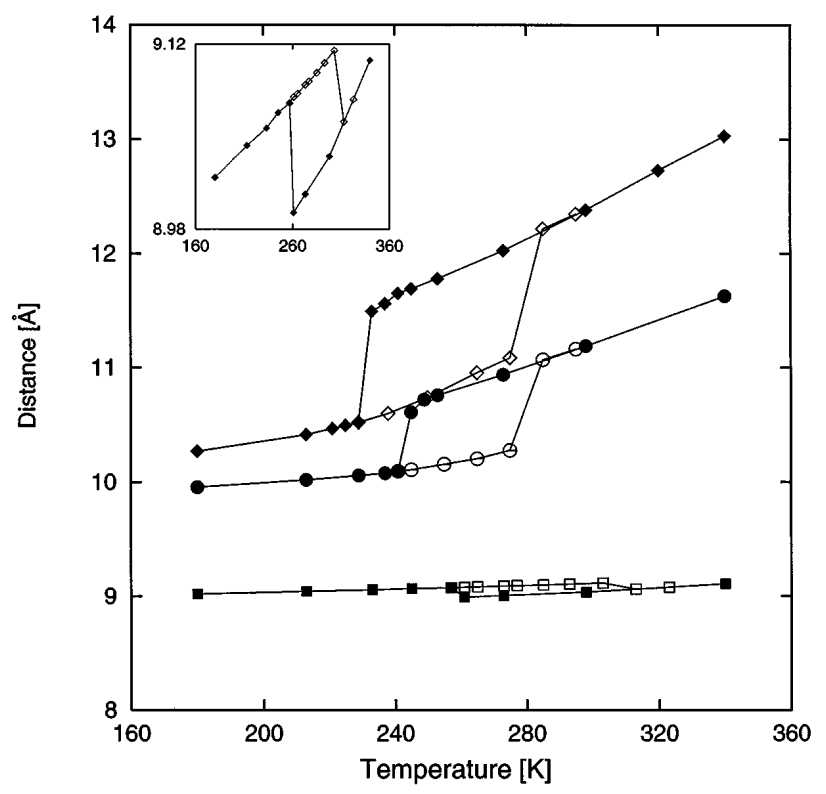

FIG. 4. Temperature dependence of the distance between two walls of the slit pore. The symbols are the same as Fig. 1. The inset is the magnification of that under a $1 \mathrm{GPa}$ load. 
from which one could build a different bilayer ice crystal, always result in the violation of the second rule to a certain extent and, consequently, bear smaller residual entropy [Fig. 3(c)] and higher potential energy.

Finally, we notice that in the freezing transition the system contracts when it is under loads of 50 and 150 $\mathrm{MPa}$, whereas it expands under $1 \mathrm{GPa}$ (see Fig. 4). This behavior is surprising in light of the fact that bulk water expands when it freezes to ice Ih. Since the difference in volume and entropy between the two phases have the same sign, when the load is low, the slope of the liquidsolid coexistence line in the $T-P_{z z}$ phase diagram should be positive. On the other hand, when the load is above $\sim 1 \mathrm{GPa}$ the slope becomes negative. For ice Ih, however, the slope is always negative.

We thank Professor D. Diestler, Dr. D. Swanson, Dr. S. Takahara, and Dr. R. Yamamoto for valuable discussions, and Dr. M. Matsumoto for help in preparing computer graphics of the ice structures. X.C.Z. thanks NSF and ONR for support of this work. H. T. is grateful to the Japan Ministry of Education and the computer center of IMS.

[1] J. D. Porter and A.S. Zinn-Warner, Phys. Rev. Lett. 73, 2879 (1994).

[2] S. Nosé, Mol. Phys. 52, 255 (1984).

[3] M. Lupkowski and F. van Swol, J. Chem. Phys. 93, 737 (1990).

[4] W. L. Jorgensen, J. Chandrasekhar, J. D. Madura, R. W. Impey, and M. L. Klein, J. Chem. Phys. 79, 926 (1983).

[5] O. A. Karim and A. D. J. Haymet, Chem. Phys. Lett. 138, 531 (1987); O. A. Karim, P. A. Kay, and A. D. J. Haymet, J. Chem. Phys. 92, 4634 (1990).

[6] H. Tanaka and I. Okabe, Chem. Phys. Lett. 259, 593 (1996).

[7] W. A. Steele, Surf. Sci. 36, 317 (1973).
[8] In the initial attempt, we performed MD simulations of 200 TIP4P molecules in a simulation cell of a square prism $\left(l_{x}=l_{y}=31 \AA\right)$ and observed a freezing transition to a bilayer ice crystal with some defects. From the initial results, we determined the simulation cell dimensions and the area density such that a crystal could be perfect (defect free) under the periodic boundary condition and satisfy the condition of $P_{x x} \sim P_{y y} \sim P_{z z}$. Moreover, preliminary simulations of a 4 times larger system $(N=768)$ with the same area density show no change of our conclusion. Note that phase behavior of a thin film of water does depend on film thickness which is controlled by the area density. For example, a thin film of water of five or six molecular layers shows no phase transition of this kind. K. Koga, X. C. Zeng, and H. Tanaka (to be published).

[9] H. Tanaka, Nature (London) 380, 328 (1996).

[10] C. A. Angell, Science 267, 1924 (1995).

[11] P. H. Poole, F. Sciortino, U. Essmann, and H. E. Stanley, Nature (London) 360, 324 (1992).

[12] C. J. Roberts, A.Z. Panagiotopoulos, and P. G. Debenedetti, Phys. Rev. Lett. 77, 4386 (1996).

[13] F. H. Stillinger, Science 225, 983 (1984).

[14] N. Materer et al., J. Phys. Chem. 99, 6267 (1995).

[15] K. M. Park, R. Kuroda, and T. Iwamoto, Angew. Chem. Int. Ed. Engl. 32, 884 (1993).

[16] M. Odelius, M. Bernasconi, and M. Parrinello, Phys. Rev. Lett. 78, 2855 (1997).

[17] At the high pressure (1.27GPa), the TIP4P bulk ice Ih is transformed to the high density amorphous ice, which is different from the bilayer ice we found [I. Okabe, H. Tanaka, and K. Nakanishi, Phys. Rev. E 53, 2638 (1996)]. Thus the TIP4P model satisfactorily reproduces properties of bulk ice in a wide range of temperature and pressure.

[18] D. Eisenberg and W. Kauzmann, in The Structure and Properties of Water (Oxford University Press, New York, 1969), p. 91.

[19] L. Pauling, J. Am. Chem. Soc. 57, 2680 (1935).

[20] L. Onsager and M. Dupuis, Rc. Scu. Int. Fis. "Enrico Fermi” 10, 294 (1960). 